\title{
Optimal Controls for a Model with Pharmacokinetics Maximizing Bone Marrow in Cancer Chemotherapy *
}

\author{
Urszula Ledzewicz ${ }^{\dagger}$ \\ Dept. of Mathematics and Statistics, \\ Southern Illinois University at Edwardsville, \\ Edwardsville, Illinois, 62026-1653, \\ uledzew@siue.edu, \\ Heinz Schättler \\ Dept. of Electrical and Systems Engineering, \\ Washington University, \\ St. Louis, Missouri, 63130-4899, \\ hms@wustl . edu
}

\begin{abstract}
A mathematical model for the depletion of bone marrow under cancer chemotherapy is analyzed as an optimal control problem. The control represents the drug dosage of a single chemotherapeutic agent and pharmacokinetic equations which model its plasma concentration are included. The drug dosages enter the objective linearly. It is shown that optimal controls are bang-bang, i.e. alternate the drug dosages at full dose with rest-periods in between, and that singular controls which correspond to treatment schedules with varying dosages at less than maximum rate are not optimal. Numerical simulations are given to illustrate the effect of the pharmacokinetic equations on the dosages.
\end{abstract}

\section{Introduction}

Although mathematical models for cancer chemotherapy have been analyzed since the early seventies, less research has been done in actually formulating them as optimal control problems [8, 24]. In these formulations the control variable represents the drug dosage, often the killing agent, and the objective captures the major goal of chemotherapy which is to maximize the killing effect on the cancer cells while at the same time keeping the same effect done to the healthy cells, mostly bone marrow cells, acceptable. The overall aim is to design an optimal, in the sense of achieving this goal, scheduling and dosage of drug treatment. Considerable research has been done in this direction analytically (e.g. [2, 3, 4, 6, 29]) as well as experimentally and clinically (e.g. $[10,17,27])$. But clearly a not so obvious optimal protocol is extremely difficult to be identified in the laboratory setting due to the essentially infinite number of cases which should be taken into account and evaluated. Optimal control theory can assist with such an analysis.

Since the drugs affect two types of cells, cancer as well as healthy, this immediately creates two possible choices in the construction of the dynamics. Most models $[6,18,20,26]$ put cancer cells as central and differentiate various stages of the cell cycle to create the dynamics of so-called compartmental models. Various models of this type were formulated and analyzed including, for example, three-compartment models with two-drug treatments where the actions of a killing drug were coupled with either blocking

\footnotetext{
*This research was supported by NSF grant DMS 0305965 and collaborative research grants DMS 0405827/0405848.

†partially supported by SIUE Hoppe Research Scholar Award and 2004 Summer Research Fellowship
} 
or recruitment agents $[26,13,14,25]$ or various models with developing stages of drug resistance. In all these models the dynamics represents the effect of the drug on the cancer cells exclusively and the damage done to bone marrow or other healthy cells are not included in the dynamics at all. The overall effect of the killing drug on these cells is only measured in the objective under a term called 'toxicity' or 'negative side effects' of the drug which often is taken as the integral of the control representing the overall amount of drug administered. On the other hand, estimates of the negative side effects of the therapeutic agents seem to be as important in the overall assessment of the drug as is its performance as a killing drug on cancerous cells. Attempts to capture both aspects in a parallel manner have been made, for example in $[20,1]$, where the problem was modelled and analyzed with a dynamics for both normal and tumor populations and with loss-functions representing the overall effects of the drugs. However, the inclusion of these two types of cells into the dynamics limits the possibility of differentiating these cells further (cancer cells at various stages of the cells cycle, bone marrow, dormant and proliferating cells) since large systems cannot easily be analyzed with the tools of optimal control theory. Like in all modelling there is a "trade-off" between trying to capture the complexities of the underlying biological processes and creating a mathematical framework that is feasible for analysis. Thus some models which focus on certain biological aspects automatically have to make other interactions and characteristics secondary.

In the mathematical model analyzed in this paper the side-effects of the drugs are central. For many drugs the limiting tissue is hemopoietic (related to blood cell formulation). Mature cells of these renewing tissues are formed through differentiation from the self-renewing stem-cell population in the bone marrow and it is generally accepted that "ideal cancer treatment would aim to bring about minimal normal stem cell kill" [11]. Toxicity to the bone marrow thus is one of the main limiting factors in chemotherapy and should be taken into account. This also is directly related to the clinical practice of taking a blood cell count of the patient before treatment sessions. If the blood cell count is too low clinicians will either delay the treatment or give a reduced dose. Thus the blood count becomes a deciding factor in designing the treatment. Therefore in this model "healthy" cells are taken as bone marrow.

The mathematical model for bone marrow proliferation under drug treatment which we consider here is based on a two-compartment growth model for tissue [8] that was considered in the nineties by Panetta [22] and analyzed as an optimal control problem by Fister and Panetta in [9] with an objective of Lagrange type that was quadratic in the control, a so-called $L^{2}$-objective. The analysis led to protocols which after an interval of no treatment were gradually increasing achieving a full dose at the terminal time of the therapy. In [15] we analyzed this model with an objective which is linear in the control, a so-called $L^{1}$-objective, and contains a terminal term representing the total count of the bone marrow cells at the end of the therapy. In this case partial doses are not optimal and in all our simulations the drug is applied in one "full-dose session" over an interval prior to the end of the therapy period.

Other important aspects that often are not taken into account in modelling cancer chemotherapy as optimal control problems are pharmacokinetics $(P K)$ and pharmacodynamics $(P D)$ of the drug modelled. In most models it is assumed that the control represents the drug dosage, which then in the dynamics and objective of the problem also plays the role of concentration and effect. This basically means that the concentration of the drug is equal to its dosage and the effects are instantaneous, a clear oversimplification. It is well known that for some cancer drugs it can take up to 24 hours until the concentration builds up and the drug starts acting on the cell. On the other hand, it is also clear that any new aspect added to the model, while making it more realistic, complicates the analysis.

In this paper we revisit the model from [15], but augment it by a pharmacokinetic $(P K)$ equation (in the form of a bilinear control system proposed by us) which models the time evolution of the drug's concentration in the body/plasma. The classical $P K$ model of exponential growth and decay is a special case of the proposed one. Pharmacodynamics $(P D)$ is still kept in its rudimentary version as it was originally modelled in [9] with the drug's effect proportional to the number of proliferating cells. It is shown that the introduction of a pharmacokinetic equation does not change the qualitative structure of the solution from [15] - partial doses still are not optimal and optimal controls alternate between chemotherapy sessions of "full dose" and rest-periods. Two types of objectives, one with the dosage, i.e. the control, the other with the concentration measuring the effect of drug on cancer cells, are introduced, but this distinction does not make a difference in the analysis. While the model is specified through 
a number of cell-cycle specific parameters, our analysis does not depend on the actual values of these parameters. Simulations are given for both models with and without pharmacokinetic equations to illustrate the quantitative changes.

\section{A Mathematical Model for Bone Marrow Depletion Under Chemotherapy}

The model for cancer chemotherapy considered below assesses the negative side-effects of chemotherapy on healthy cells which are considered as bone marrow. The effects of the drugs on cancer cells are not modelled in the dynamics, but will be taken into account indirectly in the objective for the optimal control problem.

\subsection{The dynamics of the uncontrolled model}

We briefly review the underlying model $[8$, pg. 66 ]. In the model proliferating cells $P$ and quiescent (or dormant) cells $Q$ are distinguished. The growth rate of the proliferating cells is denoted by $\gamma$ and the transition rates from proliferating to quiescent cells and vice versa are denoted by $\alpha$ and $\beta$ respectively. The rate at which bone marrow enters the blood stream is denoted by $\rho$ and the natural death rate of the proliferating cells is called $\delta$. It is assumed that all these parameters governing the cell cycle can be considered constant over the time horizon considered. Thus the overall dynamics of the uncontrolled system is described by

$$
\begin{array}{ll}
\dot{P}=(\gamma-\delta-\alpha) P+\beta Q, & P(0)=P_{0}, \\
\dot{Q}=\alpha P-(\rho+\beta) Q, & Q(0)=Q_{0},
\end{array}
$$

with all initial conditions positive.

In steady-state this corresponds to a model of exponential growth of the overall bone marrow at a fixed rate $\xi$ given by

$$
\xi=\omega \bar{x}-\rho, \quad \omega=\gamma-\delta+\rho>0,
$$

where $\bar{x}$ is the unique positive root of the quadratic equation

$$
-\omega x^{2}+(\omega-\alpha-\beta) x+\beta=0 .
$$

For, if

$$
x=\frac{P}{P+Q} \quad \text { and } \quad y=\frac{Q}{P+Q}=1-x
$$

denote the portions of the cells in the respective compartments, then $x$ satisfies the scalar Riccati equation

$$
\dot{x}=-\omega x^{2}+(\omega-\alpha-\beta) x+\beta
$$

which has a locally asymptotically stable equilibrium at $\bar{x}$ in the open interval $(0,1)$ which contains the closed interval $[0,1]$ in its region of attraction.

For our simulations we use the parameters from [9] given by $\alpha=5.643, \beta=0.48, \gamma=1.47, \delta=0$, and $\rho=0.164$. In this case we have $\bar{x}=0.1031$ and $\xi=0.0044$. In particular, in steady state only about $10 \%$ of the bone marrow cells are in their proliferating state and the total bone marrow mass is quite stagnant. Figs. 1 and 2 give the graphs of trajectories of the system for various initial conditions (the percentages of cells in the proliferating compartment are initially 10,50 and $90 \%$, respectively, with the total bone marrow cells normalized to 1 ). These simulations show how quickly the steady-state behavior is reached for the percentages. While the total number of bone marrow cells grows slowly as the steady-state is

reached, note, however, that higher initial numbers of proliferating cells produce significantly higher total numbers of bone marrow cells. The reason is the high transition rate $\alpha$ from proliferating to quiescent 
cells. Thus not only the total initial bone marrow cells, but also their distribution as proliferating and dormant cells, i.e. the initial condition of (1)-(2), determines the total number of bone marrow cells. This transition effect would not be captured in a scalar exponential growth model alone.

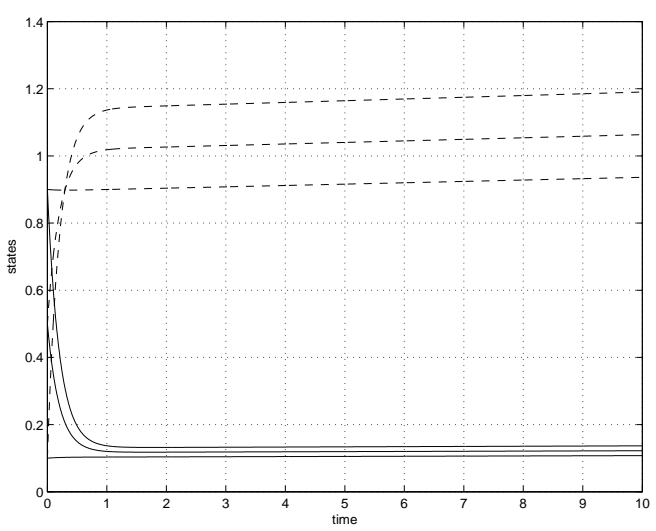

Fig. 1: Evolution of the states for the uncontrolled system

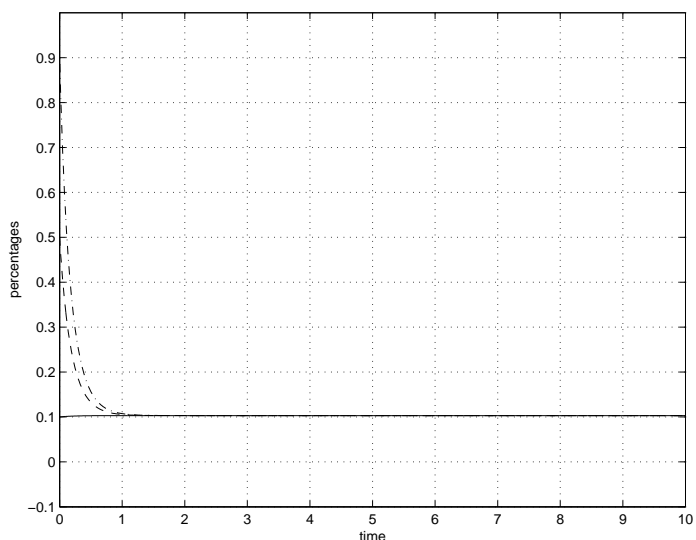

Fig. 2: Evolution of $x=\frac{P}{P+Q}$

\subsection{The controlled dynamics without pharmacokinetics}

Drug treatment is modelled by a bounded measurable function $u$ which takes values in the compact interval $[0,1]$. In the model as it was initially considered by Fister and Panetta in [9] this variable $u$ actually does not model the drug dosages, but the effects of the chemotherapeutic treatment with $u=1$ representing maximal chemotherapy and $u=0$ corresponding to no chemotherapy. While chemotherapy kills proliferating cells, it is assumed in the model that the quiescent cells are not affected by the agent. With a parameter $s>0$ to model the effectiveness of the drug as in [9], the overall dynamics can be described as

$$
\begin{array}{ll}
\dot{P}=(\gamma-\delta-\alpha-s u(t)) P+\beta Q, & P(0)=P_{0}, \\
\dot{Q}=\alpha P-(\rho+\beta) Q, & Q(0)=Q_{0} .
\end{array}
$$

If we formally assume instantaneous effects of the drug (mathematically we can think of a zero-order controller linking drug dosages to the effectiveness of the treatment) we may call $u$ the drug dosage. In this sense pharmacokinetics is assumed instantaneous and so is pharmacodynamics, but the choice of $s$ allows for variations in the effectiveness of the drugs.

If we set $N=(P, Q)$, then the general form of the dynamics is given by the bilinear system [19]

$$
\dot{N}=(A+s u B) N, \quad N(0)=N_{0},
$$

where $A$ and $B$ are fixed $(2 \times 2)$-matrices given by

$$
A=\left(\begin{array}{cc}
\gamma-\delta-\alpha & \beta \\
\alpha & -(\rho+\beta)
\end{array}\right) \quad \text { and } \quad B=\left(\begin{array}{cc}
-1 & 0 \\
0 & 0
\end{array}\right)
$$

It is easily seen that for any control $u$ the trajectory (i.e. solution to the dynamics (9) with control $u$ ) exists on all of $[0, T]$ and that each coordinate of $N(t)$ remains positive for all times $t \geq t_{0}$ (see also Proposition 1 below).

\subsection{The controlled dynamics including pharmacokinetics}

We now augment the system (7) and (8) with pharmacokinetic (PK) equations which model the time evolution of the drug's concentration in the body/plasma. Let $u$ denote the drug dosage with $u=1$ 
corresponding to a maximal dose and $u=0$ denoting no treatment. Simple models considered in the literature (for example, see $[24,20]$ ) use a first-order linear system

$$
\dot{c}=-f c+h u, \quad c(0)=0,
$$

where $f$ and $h$ are positive constants to represent the dynamics for the drug concentration $c$ in the plasma. The model itself is one of exponential growth/decay as it is commonly used as model for continuous infusions. Here we more generally propose a bilinear system of the form

$$
\dot{c}=-(f+u g) c+h u, \quad c(0)=0,
$$

with an additional parameter $g$ added. This model introduces some mild nonlinearities and allows for the feature that concentrations build up to their maximum level at a rate different from the one at which the drug is cleared by the system if no additional drugs are given. This makes sense since these are physiologically different procedures. However, in order to exclude the unrealistic scenario when the drug clears faster than it builds up we only consider the case when $g$ is non-negative. For the special case $g=0$ this reduces to the linear system (11).

The bilinear model (12) represents an attempt at introducing nonlinearities into the $P K$-model and could be replaced with more complicated nonlinear structures. (But then our analysis in section 3 below would need to be adjusted and carried out anew.) The bilinear model has the advantages that it still is a model of exponential growth or decay for constant dosages, that it allows for different rates at which the concentrations build up $(f+g)$ to their maximum level and decay $(f)$ if no drugs are given, and still the parameters are easily related to standard pharmacokinetic data: the maximum concentration is given by $c_{\max }=h /(f+g)$ which is attained asymptotically for a constant infusion $u \equiv 1$ and the parameters $f$ and $g$ are related to the times $t c_{50}$ it takes for the concentration to reach $50 \%$ effectiveness by

$$
t c_{50}^{u p}=\frac{\ln 2}{f+g} \quad \text { and } \quad t c_{50}^{\text {down }}=\frac{\ln 2}{f} .
$$

Figs. 3 and 4 illustrate the evolution of the concentration as reaction to a drug dose equal to $u=1$ on the interval from time 0 to time 2 . The maximum concentration was normalized to $c_{\max }=1$ while $t c_{50}^{u p}$ and $t c_{50}^{\text {down }}$ were varied. Note that it seems to be clear from some of the graphs in Fig. 4 that by properly choosing the parameters $f, g$, and $h$, the dynamic response can be tailored to reflect the graphs typically shown for bolus injections simply with the understanding that the time interval of application is very small.

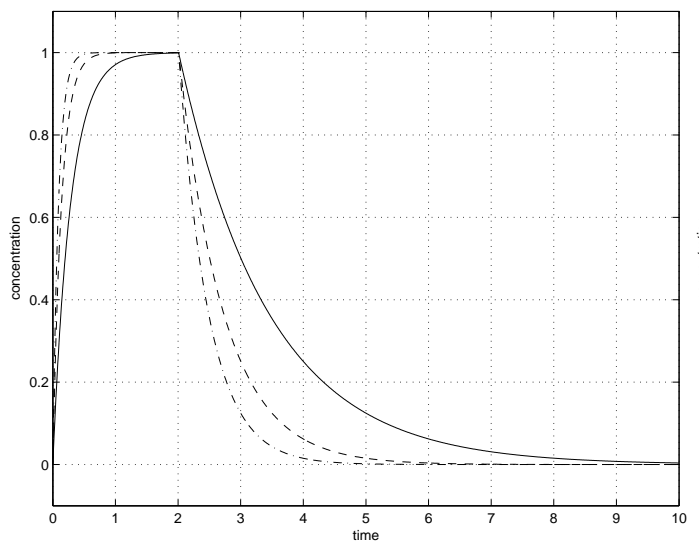

Fig. 3: $P K$ curves

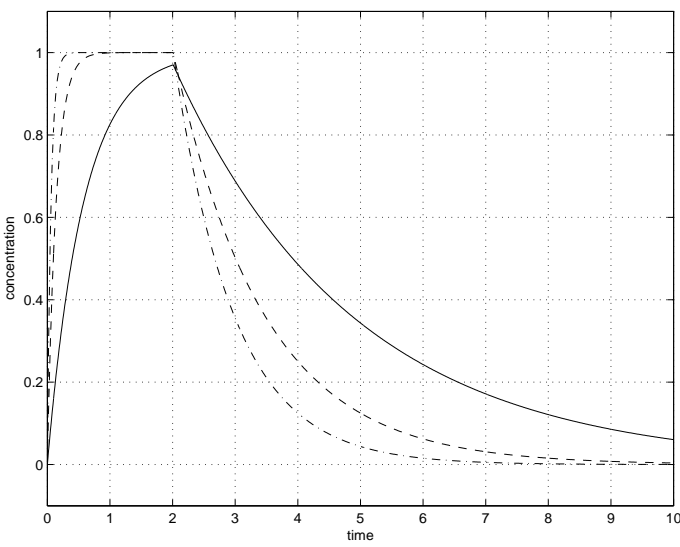

Fig. 4: $P K$ curves

Replacing the variable $u$ in (9) with the concentration $c$, we thus obtain the following system:

$$
\begin{aligned}
\dot{N} & =(A+s c B) N, & N(0) & =N_{0}, \\
\dot{c} & =-(f+u g) c+h u, & c(0) & =0 .
\end{aligned}
$$




\subsection{Objective}

The aim of any treatment is to kill the cancer or at a minimum to curtail its further spread while keeping the toxicity to the normal tissue acceptable. The objective in this model therefore becomes to give as much of the drug as possible (which will kill the cancer cells), but at the same time keep the bone marrow high. In [9] Fister and Panetta therefore maximize an objective of the form

$$
J=\int_{0}^{T} a(P(t)+Q(t))-\frac{b}{2}(1-u(t))^{2} d t \rightarrow \max
$$

over the class $\mathcal{U}$ of all Lebesgue measurable functions which take values in the control set $U=[0,1]$ a.e; $a$ and $b$ are positive constants. The use of a function which is quadratic in the control has mathematical advantages since the corresponding Hamiltonian,

$$
H=a(P(t)+Q(t))-\frac{b}{2}(1-u(t))^{2}+\lambda_{1}((\gamma-\delta-\alpha-s u(t)) P+\beta Q)+\lambda_{2}(\alpha P-(\rho+\beta) Q)
$$

will be strictly concave in the control with a unique maximum. It is shown in [9] that for $T$ sufficiently small a unique optimal control exists which is continuous on $[0, T]$. However, only at the terminal time $T$ does the optimal control take the maximum value $u=1$ and otherwise it is strictly smaller than one, $u(t)<1$ for $t<T$. In all the simulations in [9] the optimal controls are first given by $u=0$ and from a certain time on the drug dosages strictly increase to reach level 1 at the terminal time. This leads to a depletion of bone marrow towards the end which is natural since the latter values have a smaller contribution to the objective.

While the mathematical problem becomes easier with a quadratic control term in the objective, from a modelling perspective this somewhat undermines the negative effects of the drug since for example half a dose is only measured as a quarter. Thus naturally optimal solutions will have the tendency to give partial doses of the drug, if at all. An objective which is linear in the control does not provide such an incentive and as we shall see naturally leads to treatment protocols which alternate between intervals when a full dose is given and intervals where no drugs are administered, so-called bang-bang controls. In this paper we therefore consider a performance index in the form

$$
J=r N(T)+\int_{0}^{T} q N(t)+b u(t) d t \rightarrow \max
$$

where $r=\left(r_{1}, r_{2}\right)$ and $q=\left(q_{1}, q_{2}\right)$ are row-vectors with $r_{i}>0$ and $q_{i} \geq 0 ; b$ is a positive constant.

In the objective (18), as in [9], we have incorporated a term $q N(t)=q_{1} P(t)+q_{2} Q(t)$ in the Lagrangian in an effort to keep the number of bone-marrow cells high. Rather than requiring an absolute lower bound for the bone marrow, this so-called "soft" constraint implicitly maximizes the bone marrow. In addition we have added a terminal term $r_{1} P(T)+r_{2} Q(T)$ which represents a weighted average of the total bone marrow at the end of an assumed fixed therapy interval $[0, T]$ in order to prevent that the bone marrow would be depleted too much towards the end of the therapy interval. Since the aim of chemotherapy is to kill cancer cells we also want to maximize the amount of drug given which acts against the maximization of bone marrow cells. Thus the integral over the control in the objective models the cumulative effects of the treatment on the cancer, but only indirectly. It would be equally reasonable to use the concentration $c$ instead of $u$ under the integral,

$$
\hat{J}=r N(T)+\int_{0}^{T} q N(t)+b c(t) d t \rightarrow \max
$$

Analyzing both models we have seen that there is no qualitative difference in the results and thus here we only formulate the problem with objective $J$, but below we also include some simulations for $\hat{J}$.

The mathematical problem therefore can be formulated as to maximize (18) over all Lebesgue measurable functions $u$ which take values in $[0,1]$ subject to the dynamics (9) respectively (14)-(15) and 
given initial conditions. We abbreviate the corresponding optimal control problems by $\mathcal{P}_{0}$ and $\mathcal{P}_{p k}$ respectively. In [15] the problem $\mathcal{P}_{0}$ has been analyzed and (modulo some mathematical degeneracies) it has been shown that optimal controls are bang-bang, i.e. alternate between intervals of full dose and restperiods. Below we extend this analysis to the model $\mathcal{P}_{p k}$.

\section{Analysis of the Model with Pharmacokinetics}

\subsection{Necessary Conditions for Optimality}

First-order necessary conditions for optimality are given by the Pontryagin Maximum Principle [23, 5]. It can easily be shown that extremals are normal (in the sense of optimal control) and therefore these conditions reduce to the following statement: If $u_{*}$ is an optimal control with corresponding trajectory $\left(N_{*}, c_{*}\right)$, then there exists an absolutely continuous function $(\lambda, \mu)$, which we write as row-vectors $\lambda$ : $[0, T] \rightarrow\left(\mathbb{R}^{2}\right)^{*}, \mu:[0, T] \rightarrow \mathbb{R}^{*}$, satisfying the adjoint equations with transversality condition,

$$
\begin{array}{ll}
\dot{\lambda}=-\lambda(A+s c B)-q, & \lambda(T)=r, \\
\dot{\mu}=\mu(f+u g)-s \lambda B N, & \mu(T)=0,
\end{array}
$$

such that the following condition is satisfied: the optimal control $u_{*}$ maximizes the Hamiltonian

$$
H=q N+b u+\lambda(A+s c B) N+\mu(-(f+u g) c+h u)
$$

over the control set $[0,1]$ along $\left(\lambda(t), \mu(t), N_{*}(t), c_{*}(t)\right)$.

We call a pair $((N, c), u)$ consisting of an admissible control $u$ with corresponding trajectory $(N, c)$ for which there exist multipliers $(\lambda, \mu)$ such that the conditions of the Maximum Principle are satisfied an extremal (pair) and the triple $((N, c), u,(\lambda, \mu))$ is an extremal lift (to the cotangent bundle).

Optimal controls $u_{*}$ maximize the Hamiltonian $H$, i.e.

$$
(b+\mu(t)(h-g c(t))) u_{*}(t)=\max _{0 \leq u \leq 1}(b+\mu(t)(h-g c(t))) u .
$$

Thus, if we define the so-called switching function $\Phi$ by

$$
\Phi(t)=b+\mu(t)(h-g c(t)),
$$

then the optimal controls are given as

$$
u_{*}(t)=\left\{\begin{array}{ll}
1 & \text { if } \Phi(t)>0 \\
0 & \text { if } \Phi(t)<0
\end{array} .\right.
$$

In particular, since $\Phi(T)=b>0$ optimal controls always end with an interval where $u(t) \equiv 1$. A priori the control is not determined by the maximum condition at times when $\Phi(t)=0$. However, if $\Phi(t) \equiv 0$ on an open interval, then also all derivatives of $\Phi(t)$ must vanish and this may determine the control. Controls of this kind are called singular while we refer to the constant controls as bang controls. Optimal controls then need to be synthesized from these candidates through an analysis of the switching function. For example, if $\Phi(\tau)=0$, but $\dot{\Phi}(\tau) \neq 0$, then the control has a switch at time $\tau$. In order to analyze the structure of the optimal controls we therefore need to analyze the switching function and its derivatives. In this analysis the following properties of the states and multipliers are important.

Proposition 1 All states $N_{i}$ and costates $\lambda_{i}, i=1,2$, are positive over the interval $[0, T]$. The concentration $c$ is zero on some initial interval $[0, \tau]$ if no control is applied and then it is positive and the corresponding multiplier $\mu$ is negative for $t<T$. 
Proof: Clearly for any admissible control $u$ the concentration $c$ takes non-negative values and will be positive once controls are applied. It furthermore easily follows from the fact that the off-diagonal entries of the matrices $A+s c B$ are positive that the states $N_{1}(t)$ and $N_{2}(t)$ remain positive for all times: For, with some functions $\varphi$ and $\psi$ the state equations for $N$ take the form

$$
\dot{N}_{1}=\varphi(t) N_{1}+\beta N_{2}, \quad \dot{N}_{2}=\alpha N_{1}+\psi(t) N_{2},
$$

with positive initial conditions. Let $\tau=\inf \left\{t \in[0, T]: N_{1}(t) \leq 0\right\}$ and $\sigma=\inf \left\{t \in[0, T]: N_{2}(t) \leq 0\right\}$. Nothing needs to be shown if these sets are empty, so assume at least one of $\tau$ or $\sigma$ exists. Since the equations are homogeneous, $\tau \neq \sigma$, and without loss of generality assume that $\tau<\sigma$. But then $\dot{N}_{1}(\tau)=$ $\beta N_{2}(\tau)>0$ contradicting the definition of $\tau$. Similarly, since we are assuming that the $r_{i}, i=1,2$, are positive, it follows that both components $\lambda_{i}(T), i=1,2$, are positive. Let $\tau=\sup \left\{t \in[0, T]: \lambda_{1}(t) \leq 0\right\}$ and $\sigma=\sup \left\{t \in[0, T]: \lambda_{2}(t) \leq 0\right\}$. Thus $\lambda_{1}(\tau)=0$ and $\lambda_{2}(\sigma)=0$. If $\tau=\sigma$, then $\dot{\lambda}_{1}(\tau)=-q_{1} \leq 0$ and $\dot{\lambda}_{2}(\tau)=-q_{2} \leq 0$. If both $q_{1}$ and $q_{2}$ are zero, then it follows from (20) that $\lambda(t) \equiv 0$ violating the terminal conditions at time $T$. Hence, at least one of them, say $q_{1}$, is positive. But then $\lambda_{1}(t)<0$ for $t>\tau$ close to $\tau$ contradicting the definition of $\tau$. If $\tau<\sigma$, then $\lambda_{1}(\sigma)>0$ and thus $\dot{\lambda}_{2}(\sigma)=-\lambda_{1}(\sigma) \beta-q_{2}<0$ and again $\lambda_{2}$ is negative for times $t>\sigma$ contradicting the definition of $\sigma$. Similarly, if $\tau>\sigma$, then $\dot{\lambda}_{1}(\tau)=-\lambda_{2}(\tau) \alpha-q_{1}<0$ leading to the same contradiction. In particular, it thus follows that

$$
\lambda(t) B N(t)=\left(\lambda_{1}(t), \lambda_{2}(t)\right)\left(\begin{array}{cc}
-1 & 0 \\
0 & 0
\end{array}\right) N(t)=-\lambda_{1}(t) N_{1}(t)<0 .
$$

Hence, $\dot{\mu}(\tau)>0$ whenever $\mu(\tau)=0$. Since $\mu(T)=0$, this implies that $\mu$ is negative for all earlier times.

\subsection{Singular extremals}

Assume the control $u$ is singular on some open interval $I$, i.e. the switching function $\Phi$ vanishes on $I$. In this case the maximum condition (23) does not determine the value of the control. Instead singular controls can be computed by differentiating the switching function in time until the control variable explicitly appears in the derivative, say in $\Phi^{(r)}(t)$, and then solving the resulting equation $\Phi^{(r)}(t) \equiv 0$ for the control. If the corresponding control value is admissible, i.e. has a value between 0 and 1 , this defines the singular control. Otherwise the singular arc is not admissible. For a single-input system which is linear in the control it is well-known [12] that $r$ must be even, say $r=2 k$, and $k$ is called the order of the singular arc. In principle, this order can vary with time over the interval $I$. If it is constant on the interval $I$, then it is a necessary condition for optimality of a singular arc of order $k$, the so-called generalized Legendre-Clebsch condition $[12,5]$, that

$$
(-1)^{k} \frac{\partial}{\partial u} \frac{d^{2 k}}{d t^{2 k}} \frac{\partial H}{\partial u} \leq 0
$$

along the extremal. Note that the term $\frac{\partial H}{\partial u}=\Phi$ in (27) represents the switching function for the problem.

For the problem $\mathcal{P}_{p k}$ we have $\Phi(t)=b+\mu(t)(h-g c(t))$ and thus by a direct computation

$$
\dot{\Phi}(t)=\mu(t) f h-s \lambda(t) B N(t)(h-g c(t)) \equiv 0
$$

on $I$. Since only the time-derivatives of $\mu$ and $c$ explicitly depend on the control, we get

$$
\begin{aligned}
\frac{\partial}{\partial u} \frac{d^{2}}{d t^{2}} \frac{\partial H}{\partial u} & =\frac{\partial}{\partial u} \ddot{\Phi}(t)=\left(\frac{\partial \dot{\mu}}{\partial u}(t)\right) f h+s \lambda(t) B N(t) g\left(\frac{\partial \dot{c}}{\partial u}(t)\right) \\
& =\mu(t) g f h+s \lambda(t) B N(t) g(h-g c(t)) .
\end{aligned}
$$

Since $\dot{\Phi}$ vanishes identically on $I$, by (28) we have that

$$
\mu(t) f h \equiv s \lambda(t) B N(t)(h-g c(t))
$$


and thus

$$
\frac{\partial}{\partial u} \frac{d^{2}}{d t^{2}} \frac{\partial H}{\partial u}=2 \mu(t) g f h
$$

If $g \neq 0$ this quantity does not vanish and the singular control is of order 1 over the full interval $I$. However, the generalized Legendre-Clebsch condition requires that $g<0$ for the singular control to be maximal. This would correspond to the unrealistic situation where the concentration clears faster for $u=0$ than it builds up for $u=1$ and this case was excluded in our assumption that $g \geq 0$.

The case $g=0$ is different since now also $\ddot{\Phi}(t) \equiv 0$ on the interval $I$. In this case the relations above simplify to

$$
\begin{aligned}
& \Phi(t)=b+\mu(t) h \equiv 0, \\
& \dot{\Phi}(t)=\dot{\mu}(t) h=(\mu(t) f-s \lambda(t) B N(t)) h \equiv 0, \\
& \ddot{\Phi}(t)=-s h \frac{d}{d t}(\lambda(t) B N(t)) \equiv 0 .
\end{aligned}
$$

For a general constant matrix $M$, it follows by direct differentiation that the derivative of $\Psi(t)=$ $\lambda(t) M N(t)$, along a solution $N$ to the system equation (14) for control $u$ and a corresponding solution $\lambda$ of the corresponding adjoint equation (20) is given by

$$
\dot{\Psi}(t)=\lambda(t)[A+s c(t) B, M] N(t)-q M N(t),
$$

where $[X, M]$ denotes the commutator of the matrices $X$ and $M$ defined as $[X, M]=M X-X M$. For,

$$
\begin{aligned}
\dot{\Psi}(t) & =\dot{\lambda}(t) M N(t)+\lambda(t) M \dot{N}(t) \\
& =(-\lambda(t)(A+s c(t) B)-q) M N(t)+\lambda(t) M(A+s c(t) B) N(t) \\
& =\lambda(t)[A+s c(t) B, M] N(t)-q M N(t) .
\end{aligned}
$$

Thus

$$
\ddot{\Phi}(t)=-\operatorname{sh}(\lambda(t)[A, B] N(t)-q B N(t)) \equiv 0
$$

and

$$
\Phi^{(3)}(t)=-s h(\lambda(t)[A+s c B,[A, B]] N(t)-q[A, B] N(t)-q B(A+s c B) N(t)) \equiv 0 .
$$

Again, since the control $u$ only appears explicitly in the derivative of $c$ in these terms, we get

$$
\begin{aligned}
\frac{\partial}{\partial u} \frac{d^{4}}{d t^{4}} \frac{\partial H}{\partial u} & =-s^{2} h\left(\frac{\partial \dot{c}}{\partial u}(t)\right)\left(\lambda(t)[B,[A, B]] N(t)-q B^{2} N(t)\right) \\
& =-s^{2} h^{2}\left(\lambda(t)[B,[A, B]] N(t)-q B^{2} N(t)\right) .
\end{aligned}
$$

Since $B^{2}=-B$ we obtain from (35) that

$$
-q B^{2} N(t)=q B N(t)=\lambda(t)[A, B] N(t)
$$

and thus

$$
\frac{\partial}{\partial u} \frac{d^{4}}{d t^{4}} \frac{\partial H}{\partial u}=-s^{2} h^{2} \lambda(t)([B,[A, B]]+[A, B]) N(t) .
$$

Direct simple computations verify that

$$
[A, B]=\left(\begin{array}{cc}
0 & -\beta \\
\alpha & 0
\end{array}\right) \quad \text { and } \quad[B,[A, B]]=\left(\begin{array}{cc}
0 & -\beta \\
-\alpha & 0
\end{array}\right)
$$

and thus

$$
\frac{\partial}{\partial u} \frac{d^{4}}{d t^{4}} \frac{\partial H}{\partial u}=-s^{2} h^{2} \lambda(t)\left(\begin{array}{cc}
0 & -2 \beta \\
0 & 0
\end{array}\right) N(t)=2 s^{2} h^{2} \beta \lambda_{1}(t) N_{2}(t)>0
$$

again violating the generalized Legendre-Clebsch condition (27) for singular controls of order 2. Thus if $g \geq 0$ then in fact singular arcs locally minimize the objective. In particular we have 
Proposition 2 Singular controls are not optimal.

For the case $g=0$ a comparison of the argument with the calculations for problem $\mathcal{P}_{0}$ in [15] shows that the addition of a first order linear controller between the drug dosage and the concentration does not alter optimality properties of singular arcs. The only change is that the order of the singular arc which is 1 for problem $\mathcal{P}_{0}$ becomes 2 for problem $\mathcal{P}_{p k}$. However, the deciding computations reduce to the same expressions.

\subsection{Bang-bang controls}

This then leaves bang-bang controls as the prime candidates for optimality and it is not difficult to compute bang-bang extremals numerically. In [15] we have formulated an algorithm which allows to determine the local optimality of the corresponding bang-bang controls for the problem $\mathcal{P}_{0}$. This algorithm is based on earlier work (see, for example $[21,13,25]$ ) and needs to be modified slightly for problem $\mathcal{P}_{p k}$ to allow for the incorporation of the pharmacokinetic equation (15). The argument itself is a straightforward application of the general results derived in [21] to the specific equations of this model. The formulation, however, gets lengthy and does not offer more insight than the discussion given in [15]. It is therefore not included here. But these results are relevant in the sense that they allow to establish the actual local optimality of numerically computed bang-bang controls. They were employed to verify the strong local optimality of all the simulations given in section 4 .

\section{Simulations and Comparisons}

Using a version of the gradient method for the calculation of extremal bang-bang controls developed earlier by Duda [7], we ran simulations for the models $\mathcal{P}_{0}$ and $\mathcal{P}_{p k}$ presented here for a therapy interval of length $T=10$ with the following parameter values taken from [9]: $\alpha=5.643, \beta=0.48, \gamma=1.47, \delta=0$, and $\rho=0.164$. In the simulations we report on below we set $s=1, r_{1}=r_{2}=1$, and also $q_{1}=q_{2}=1$. We do vary the parameter $b$ multiplying the control in the objective. For the model $\mathcal{P}_{p k}$ in addition we chose $f=5 \ln 2=3.4657, g=4 f$ and $h=f+g$. For these parameter for $u=0$ the concentration decays by $50 \%$ in $t=0.2$ time-units and for $u=1$ the concentration builds up 5 times faster. The maximum concentration is normalized to 1 .

For $b=1$ and initial conditions $\left(p_{0}, q_{0}\right)$ chosen as the steady-state of the uncontrolled system, with these parameters the optimal control is $u \equiv 1$ for both $\mathcal{P}_{0}$ and $\mathcal{P}_{p k}$. The corresponding graphs of the states are virtually indistinguishable. As the initial conditions are changed to $\left(p_{0}, q_{0}\right)=(.5, .5)$, the control has one switch from $u=0$ to $u=1$ which occurs at $\tau_{0}=0.54$ for $\mathcal{P}_{0}$ and slightly earlier at $\tau_{p k}=0.49$ for $\mathcal{P}_{p k}$. Even as the initial conditions are changed to $\left(p_{0}, q_{0}\right)=(.9, .1)$, the switchings in the control still happen quickly at $\tau_{0}=0.80$ for $\mathcal{P}_{0}$ and slightly earlier at $\tau_{p k}=0.76$ for $\mathcal{P}_{p k}$. The controls (and switching functions as dashed lines) for this simulation are given in Figs. 5 and 7 and the corresponding states in Figs. 6 and 8. The dashed line in the graphs of the states gives the evolution of the cells in the quiescent compartment while the regular line gives the evolution of the cells in the proliferating stage. In all these cases for $u \equiv 0$ the system quickly settles into steady-state and then the optimal control becomes $u \equiv 1$ for the remaining time. This behavior changes only if a different weight is used for the control in the objective. 


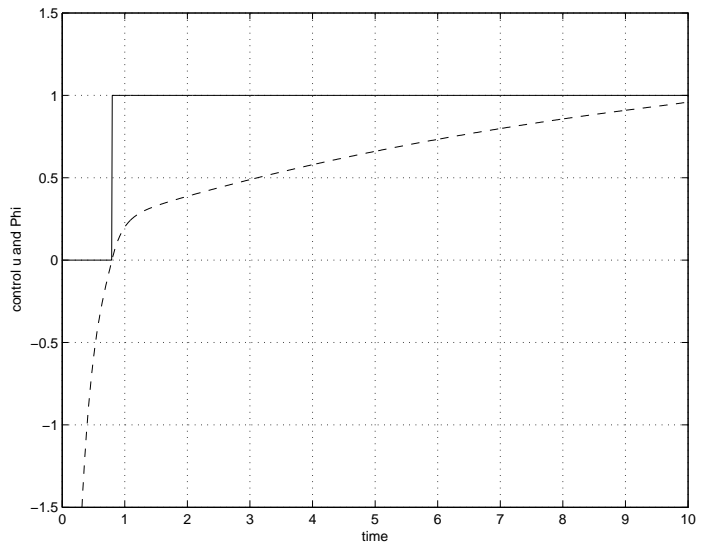

Fig. 5: Control for $p_{0}=0.9$ for $\mathcal{P}_{0}$

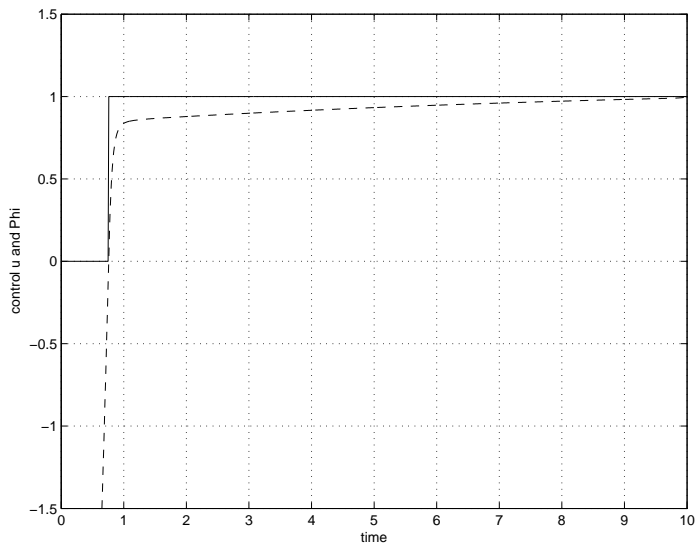

Fig. 7: Control for $p_{0}=0.9$ for $\mathcal{P}_{p k}$

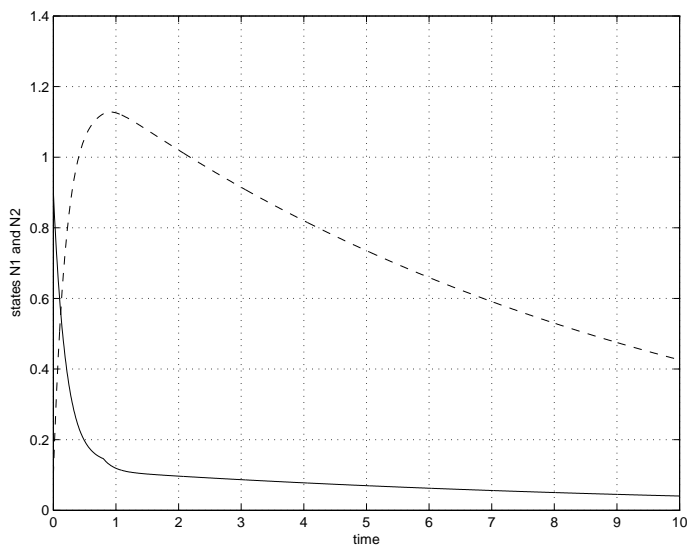

Fig. 6: States for $p_{0}=0.9$ for $\mathcal{P}_{0}$

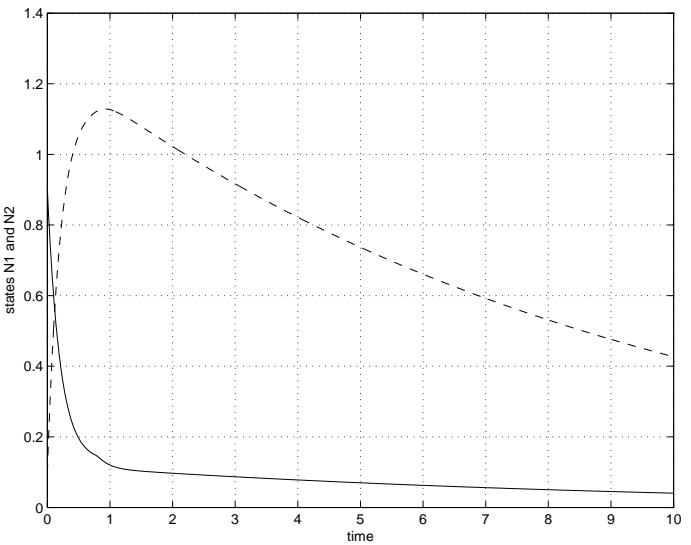

Fig. 8: States for $p_{0}=0.9$ for $\mathcal{P}_{p k}$

If the weight $b$ at the integral of the control is decreased, trajectories in these simulations still have exactly one switch from $u=0$ to $u=1$, but now the switches occur much later. For initial conditions corresponding to the uncontrolled steady-state the switchings now are at $\tau_{0}=5.02$ for $\mathcal{P}_{0}$ and again earlier at $\tau_{p k}=4.16$ for $\mathcal{P}_{p k}$. Figs. 9-12 below give the graphs for steady-state initial conditions and Figs. 13-16 give the graphs for $\left(p_{0}, q_{0}\right)=(.9, .1)$. Figs. 17-18 show corresponding graphs for the case when the problem of maximizing the objective $\hat{J}$ in (19) is considered for these parameter values. Although the controls have the same qualitative form, notice the differences in the shape of the switching functions for $\mathcal{P}_{0}$ and for the models $\mathcal{P}_{p k}$ (for maximizing $J$ respectively $\hat{J}$ ). 


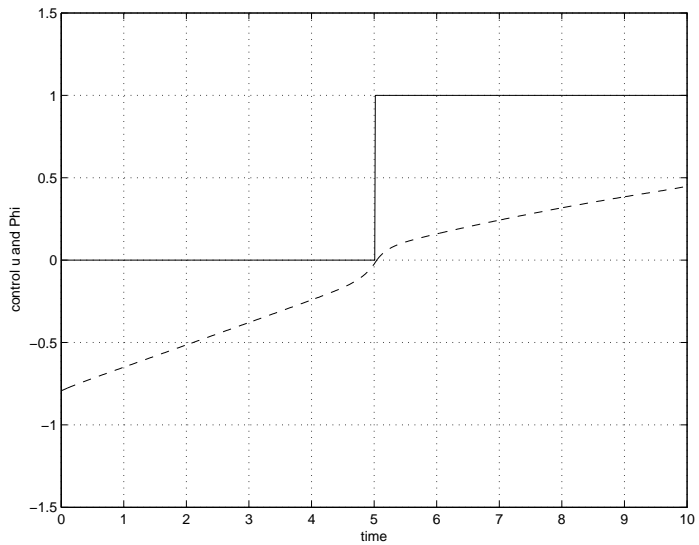

Fig. 9: Control from steady-state for $\mathcal{P}_{0}$

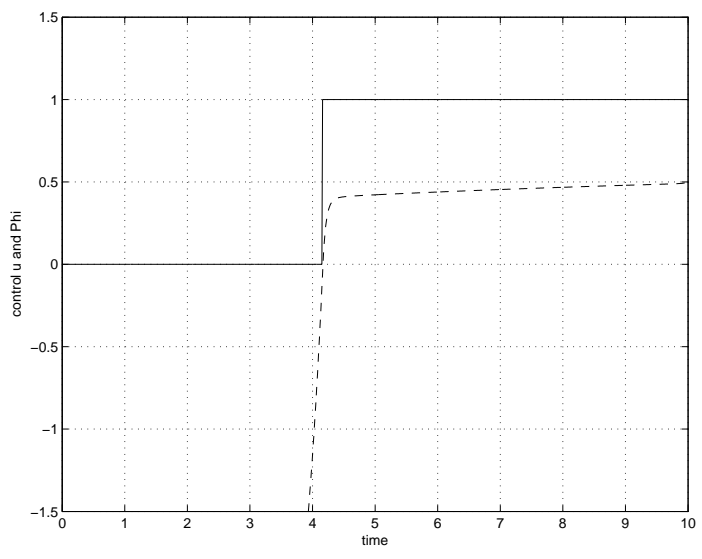

Fig. 11: Control from steady-state for $\mathcal{P}_{p k}$

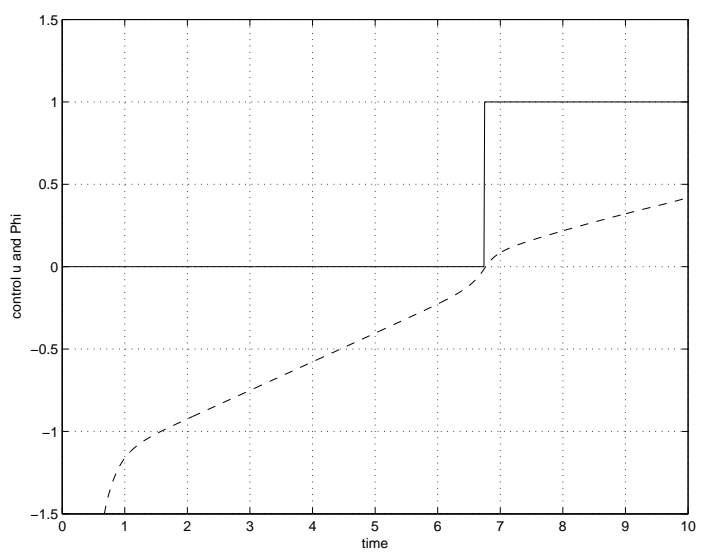

Fig. 13: Control for $p_{0}=0.9$ for $\mathcal{P}_{0}$

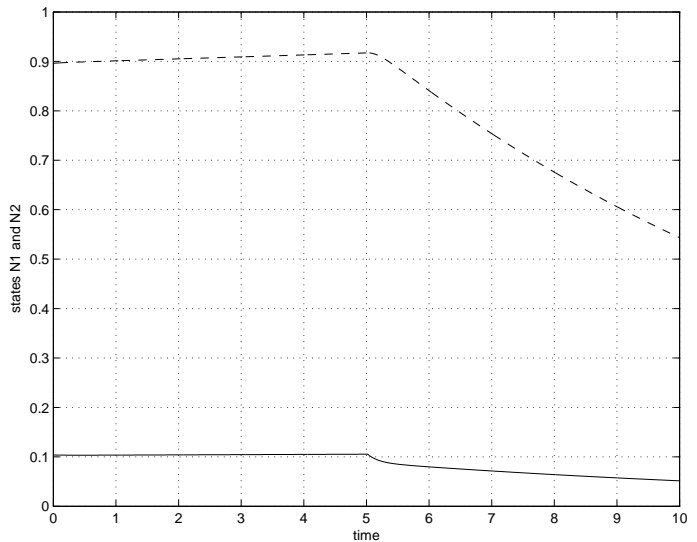

Fig. 10: States from steady-state for $\mathcal{P}_{0}$

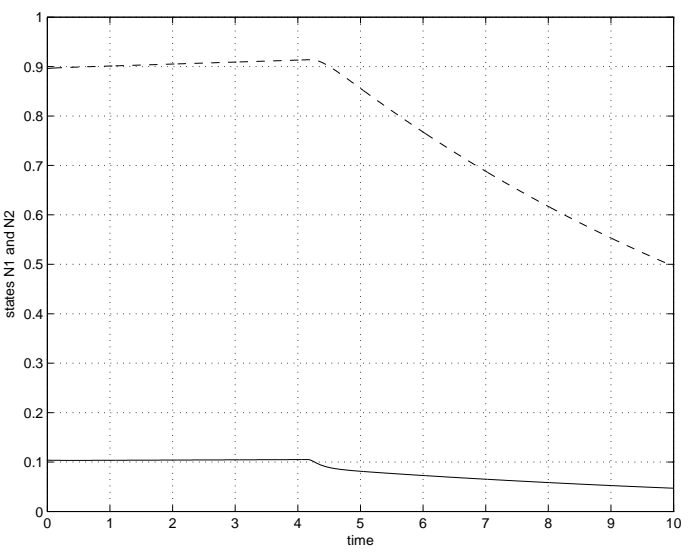

Fig. 12: States from steady-state for $\mathcal{P}_{p k}$

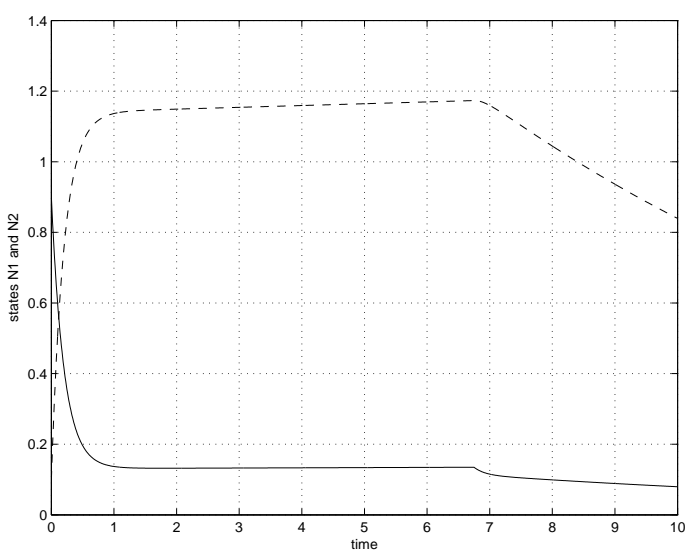

Fig. 14: States for $p_{0}=0.9$ for $\mathcal{P}_{0}$ 


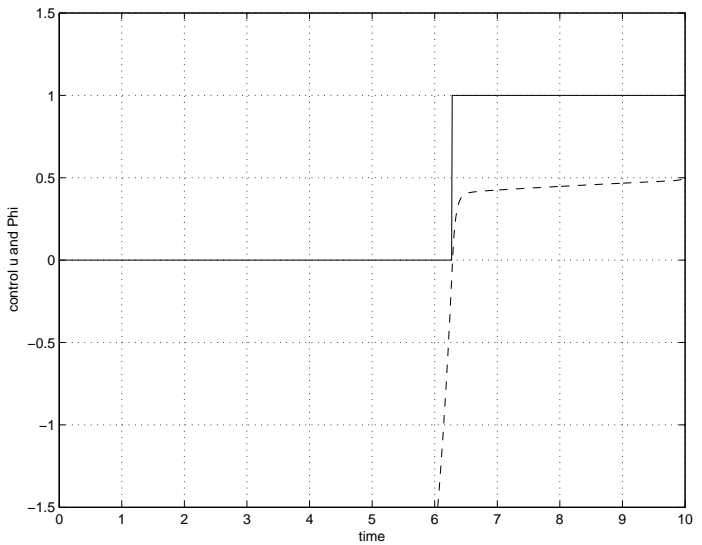

Fig. 15: Control for $p_{0}=0.9$ for $\mathcal{P}_{p k}$

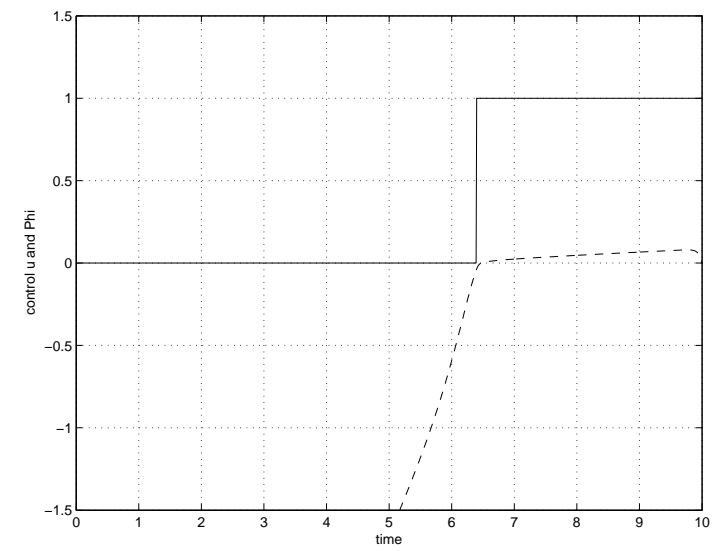

Fig. 17: Control for maximizing $\hat{J}$ for $p_{0}=0.9$

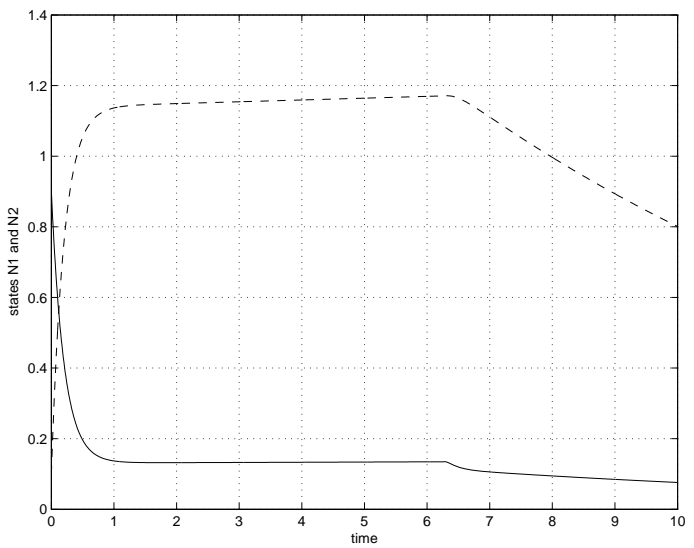

Fig. 16: States for $p_{0}=0.9$ for $\mathcal{P}_{p k}$

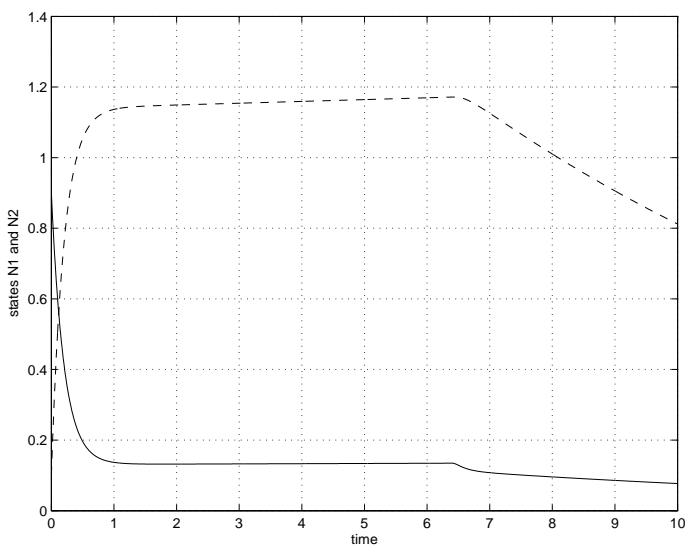

Fig. 18: States for maximizing $\hat{J}$ for $p_{0}=0.9$

In these simulations optimal controls only exhibit one switch from $u=0$ to $u=1$. The controls switch slightly earlier for the model with pharmacokinetics in an effort to compensate for the delay. If the time it takes for the concentrations to build up is made longer, then these differences become more pronounced. For example, for $f=\ln 2=0.6931, g=4 f$ and $h=f+g$ and initial conditions in steady-state for $b=.5$ the switching occurs at $\tau_{p k}=3.54$ instead of at 4.16 .

\section{Conclusion}

In this paper we analyzed a model for cancer chemotherapy that aims at minimizing the damage done to bone marrow cells during the chemotherapy. In the objective a linear term in the drug dosage was used and a pharmacokinetic equation (in form of a bilinear control system) which models the time evolution of the drug's concentration in the body/plasma was included. Pharmacodynamics was kept in its rudimentary version with the drug's effect proportional to the number of proliferating cells as it was originally modelled in [9]. The mathematical analysis shows that the introduction of a pharmacokinetic equation as exponential growth and decay does not change the qualitative structure of the solution partial doses still are not optimal and in principle optimal controls alternate between chemotherapy sessions of "full dose" and rest-periods. While the model is specified through a number of cell-cycle specific parameters, our analysis does not depend on the actual values of these parameters and thus this 
conclusion is generally valid. In fact, even if the parameters in the model are allowed to vary in time, it can be shown that singular controls are not optimal. Comparison of simulations shows that more drug is given for the models which include $P K$ which, since the concentration is directly related to the negative effects on the bone marrow in the model, is natural in view of the time delay it now takes for the concentration to build up. The quantitative changes in the switchings in our simulations are small, but this clearly depends on how fast the $P K$ reactions are. Although all simulations exhibited only one switching, there does not seem to be a straightforward way of proving such a result analytically. Convexity properties of the switching functions seen in some simulations (especially if we set $q=0$ ) in principle allow for more switchings. But this also strongly will depend on the parameters chosen in the objective. A more important change in the model is the inclusion of pharmacodynamics where the effects of the drug concentration on the bone marrow and/or cancer cells are modelled through a more realistic function $s=s(c)$. If this function saturates at certain upper and lower concentrations, then indeed singular controls can become optimal as shown in [16].

Acknowledgement. We would like to express our thanks to the Mathematical Biosciences Institute of The Ohio State University for hosting us at a workshop on "Cell Proliferation and Cancer Chemotherapy" that exposed us to some of the material in this paper.

\section{References}

[1] E.K. Afenya, Recovery of normal hemopoiesis in disseminated cancer therapy - a model, Mathematical Biosciences, 172, (2001), pp. 15-32

[2] Z. Agur, The effect of drug schedule on responsiveness to chemotherapy, Ann. New York Acad. Sci., 504, (1986), pp. 274-277.

[3] Z. Agur, R. Arnon and B. Schechter, Reduction of cytotoxicity to normal tissues by new regimes of cell-cycle phase-specific drugs, Math. Biosci., 92, (1988), pp. 1-15.

[4] L. Cojocaru and Z. Agur, A theoretical analysis of interval drug dosing for cell-cycle-phase-specific drugs, Mathematical Biosciences, 109, (1992), pp. 85-97.

[5] A.E. Bryson and Y.C. Ho, Applied Optimal Control, Hemisphere Publishing, 1975

[6] B.F. Dibrov, A.M. Zhabotinsky, Yu.A. Neyfakh, M.P. Orlova and L.I. Churikova, Mathematical model of cancer chemotherapy. Periodic schedules of phase-specific cytotoxic-agent administration increasing the selectivity of Therapy, Mathematical Biosciences, 73, (1985), pp. 1-31

[7] Z. Duda, A gradient method for application of chemotherapy protocols, J. of Biological Systems, 3, (1995), pp. 3-11

[8] M. Eisen, Mathematical Models in Cell Biology and Cancer Chemotherapy, Lecture Notes in Biomathematics, Vol. 30, Springer Verlag, (1979)

[9] K.R. Fister and J.C. Panetta, Optimal control applied to cell-cycle-specific cancer chemotherapy, SIAM J. Applied Mathematics, 60, (2000), pp. 1059-1072

[10] J.D. Hainsworth and F.A. Greco, Paclitaxel administered by 1-hour infusion, Cancer, 74, (1994), pp. $1377-1382$

[11] B.T. Hill, Cancer chemotherapy. The relevance of certain concepts of cell cycle kinetics, BBB (Rev. Canc.), 516, (1978), pp. 389-417

[12] A. Krener, The high-order maximal principle and its application to singular controls, SIAM J. Control and Optimization, 15, (1977), pp. 256-293 
[13] U. Ledzewicz and H. Schättler, Optimal bang-bang controls for a 2-compartment model in cancer chemotherapy, J. of Optimization Theory and Applications - JOTA, 114, (2002), pp. 609-637

[14] U. Ledzewicz and H. Schättler, Analysis of a cell-cycle specific model for cancer chemotherapy, J. of Biological Systems, 10, (2002), pp. 183-206

[15] U. Ledzewicz and H. Schättler, Controlling a model for bone marrow dynamics in cancer chemotherapy, Mathematical Biosciences and Engineering, 1, (2004), pp. 95-110

[16] U. Ledzewicz and H. Schättler, The influence of $P K / P D$ on the structure of optimal controls in cancer chemotherapy models, Mathematical Biosciences and Engineering, 3, (2005), to appear

[17] M.M. Lopes, E.G. Adams, T.W. Pitts and B.K. Bhuyan, Cell kill kinetics and cell cycle effects of Taxol on human and hamster ovarian cell lines, Cancer Chemother. Pharmacol., 32, (1993), pp. $235-242$

[18] R.B. Martin, Optimal control drug scheduling of cancer chemotherapy, Automatica, 28, (1992), pp. $1113-1123$

[19] R.R. Mohler, Bilinear Control Systems, Academic Press, (1973)

[20] J.M. Murray, Optimal drug regimens in cancer chemotherapy for single drugs that block progression through the cell cycle, Mathematical Biosciences, 123, (1994), pp. 183-193

[21] J. Noble and H. Schättler, Sufficient conditions for relative minima of broken extremals in optimal control theory, J. of Mathematical Analysis and Applications, 269, (2002), pp. 98-128

[22] J.C. Panetta, A mathematical model of breast and ovarian cancer treated with paclitaxel, Mathematical Biosciences, 146 (1997), pp. 83-113

[23] L.S. Pontryagin, V.G. Boltyanskii, R.V. Gamkrelidze and E.F. Mishchenko, The Mathematical Theory of Optimal Processes, MacMillan, New York, (1964)

[24] G.W. Swan, Role of optimal control in cancer chemotherapy, Mathematical Biosciences, 101, (1990), pp. $237-284$

[25] A. Swierniak, U. Ledzewicz and H. Schättler, Optimal control for a class of compartmental models in cancer chemotherapy, Int. J. of Applied Mathematics and Computer Science, 13, (2003), pp. 101-112

[26] A. Swierniak, A. Polanski and M. Kimmel, Optimal control problems arising in cell-cycle-specific cancer chemotherapy, Cell Proliferation, 29, (1996), pp. 117-139

[27] W.W. Ten Bokkel Huinink, E. Eisenhauser and K. Swenerton, Preliminary evaluation of a multicenter randomized study of TAXOL (paclitaxel) dose and infusion length in platinum-treated ovarian cancer, Cancer Treatment Rev., 19, (1983), pp. 79-86.

[28] G.F. Webb, Resonance phenomena in cell population chemotherapy models, Rocky Mountain J. of Mathematics., 20, (1990), pp. 1195-1216.

[29] G.F. Webb, Resonance in periodic chemotherapy scheduling, in: Proceedings of the First World Congress of Nonlinear Analysts, Vol.4, V. Lakshmikantham, ed., Walter DeGruyter, Berlin, 1995, pp. 3463-3747. 